\title{
Special issue on Modeling of Reactive Systems
}

\author{
Claude Martinez • Olivier H. Roux
}

Received: 7 May 2011 / Accepted: 14 May 2011 / Published online: 7 June 2011

(C) Springer Science+Business Media, LLC 2011

This Special Issue of J-DEDS on Modeling of Reactive Systems was inspired by the seventh edition of the French-speaking conference "Modélisation des Systèmes Réactifs - MSR 2009." MSR 2009 was organized by the IRCCyN and held at the École Centrale of Nantes, France, on 16-18 November 2009. MSR is a conference that traditionally gathers French-speaking researchers from both the Computer Science and the Automatic Control communities, and that are involved in the modeling, analysis and control of reactive systems.

The articles presented at MSR 2009 covered a wide range of topics, from performance evaluation of telecommunication channels to supervisory control, from simulation and test of models to timed systems analysis and control. Following the MSR 2009 conference, the MSR Steering Committee invited a small number of authors who attended MSR 2009 to submit full journal papers for publication in a special issue of J-DEDS on the topic of Modeling of Reactive Systems. These invitations reflected the variety of problems related to the study of discrete event systems that were discussed at MSR 2009. The submitted papers followed the standard editorial process of J-DEDS under the coordination of the Guest Editors and of Department Editor Stéphane Lafortune. In the end, four papers were selected for inclusion in this special issue.

The article from Thomas Ferrandiz, Fabrice Francès and Christian Fraboul presents a method to compute the worst-case delays of communications in the specific framework of SpaceWire networks. SpaceWire is a high-speed, lowconsumption network used by the European Space Agency, designed for the purpose of being used as the sole on-board network in satellites. Therefore, it has to carry both payload and control traffic on the same links. The aim is then to

C. Martinez ( $\square)$

LUNAM Université, Université de Nantes, IRCCyN UMR CNRS 6597, Nantes, France e-mail: Claude.Martinez@univ-nantes.fr

O. H. Roux

LUNAM Université, École Centrale de Nantes, IRCCyN UMR CNRS 6597, Nantes, France 
guarantee the strict time constraints associated with the control traffic. The authors have designed a method to compute an upper-bound on the worst-case end-to-end transmission delay of a packet across a SpaceWire network. The proposed approach has been tested on a network architecture designed for an observation satellite.

The article from Gabriel Kalyon, Tristan Le Gall, Thierry Massart and Hervé Marchand presents a decentralized control approach to deal with infinite systems under partial observations. The paper reports their work on the state avoidance decentralized control problem for infinite discrete event systems modeled by Symbolic Transition Systems (STS). The authors provide an algorithm that overcomes the complexity of such problems with the help of an over-approximation technique that leads to an effective computation of controllers. Although the controllers obtained by this method meet the specifications, they may forbid more states than needed.

The paper proposed by Florent Peres, Bernard Berthomieu and François Vernadat tackles the problem of time Petri nets composition. The article deals with the building of complex systems in a compositional manner from components specified by time Petri nets (TPN). The authors propose a first solution that is adequate for a particular subclass of Time Petri nets but that significantly increases the complexity of components. Then an improved solution is developed, based on an extension of time Petri nets with two relations added on the transitions. This latter solution requires a much simpler transformation of nets, does not significantly increase their complexity and is applicable to a larger class of TPN.

The last paper presents the work of Frédéric Boniol, Mikel Cordovilla, Julien Forget and Claire Pagetti on multi-task synchronous program implementation. It provides a complete scheme for the integration and the development of multiperiodic critical embedded systems. The formal description of the real-time software architecture of a multi-periodic systems is translated into a multi-threaded code executable with an on-line priority-based scheduler. The translation is formally proved as correct, meaning that the generated code respects the real-time semantics of the original program (respect of periods, deadlines, release dates and precedences) as well as its functional semantics (respect of variable consumption).

We would like to express our gratitude towards the authors for their contributions and timely revisions, to the reviewers for their work and constructive suggestions that contributed to the quality of the final articles, and also to the editorial board of J-DEDS for giving us the opportunity to present this selection of contributions. 